\title{
Incidental learning of event frequency
}

\author{
ROBERT L. GREENE \\ Yale University, New Haven, Connecticut
}

\begin{abstract}
Two experiments studied the effect of intentionality of learning on memory for the frequency of occurrence of words on a list. Subjects who learned the items intentionally in preparation for an unspecified memory test remembered frequency as accurately as did those who studied specifically for a frequency-estimation test. Both groups recalled frequency information more accurately than did a group that learned the words incidentally. These results, along with a review of the literature, suggest that there is no strong evidence for the automatic encoding of frequency information.
\end{abstract}

There has been considerable interest in recent years in automatic processes, that is, processes that occur without intention or effort. Hasher and Zacks (1979; Zacks, Hasher, \& Sanft, 1982) have suggested that one such process is the encoding of frequency information. In other words, they have proposed that people automatically learn how often an event has occurred.

Hasher and Zacks (1979) proposed a set of five criteria for determining whether frequency information is encoded automatically. This paper is concerned with one of those criteria: Intentionality of learning, or instructional set, should have no effect on accuracy in a frequency-estimation test. It is typically found (Flexser \& Bower, 1975; Howell, 1973; Zacks et al., 1982) that subjects who know that they will be tested on the frequency of the items on a list do no better on a frequency test than do subjects who are told only to expect either an unspecified "memory test on the items" or a free-recall test.

Although these studies are often cited as evidence for the automatic encoding of frequency information, they may be interpreted in other ways. For example, it is possible that people do not know any effective way to keep track of the frequencies of a very large number of items. Warning them that they will be tested on frequency would therefore do them no good. It is quite possible that, if they were told an effective strategy to follow, their performance would improve (see Zacks et al., 1982, Experiment 2). Alternatively, even subjects who are expecting only an unspecified "memory test" on the items might adopt a mnemonic strategy that involves keeping track of the frequency of the items.

Several investigators (Lehman, 1982; Mandler, Seegmiller, \& Day, 1977) have proposed that the es-

This research was supported by NSF Grant BNS 8005838 to R. Crowder. I am very grateful to Robert Crowder and James Nairne for their help with this research. Requests for reprints should be addressed to Robert L. Greene, Department of Psychology, Yale University, Box 11A Yale Station, New Haven, Connecticut 06520 . sential test for determining automatic processing of an attribute of an item is between incidental and intentional learning of the items themselves, as opposed to varying the intent to learn that specific attribute. The crucial comparison is between one group of subjects who know that they will be tested on the items and a second group that learns the items incidentally and would have no reason to encode a particular attribute. The first experiment in this paper applied this comparison to frequency information. Subjects were told either that they would not be tested at all on the words (incidental learning) or that their "memory for the words" would be tested (intentional learning). All subjects were then tested on the frequency of the items.

A somewhat similar experiment was performed by Rose and Rowe (1976). In that study, it was shown that an incidental-learning group of subjects who had to perform a semantic orienting task for each word remembered frequency as well as did subjects who were told either that they would be tested on the words or that they would be given a test on the frequency of the words. These latter two groups of subjects did not have to perform any orienting activity on the words. This ex. periment left unanswered the question of whether incidental-learning subjects who do not have to process the items semantically would also do as well as would subjects in these other conditions. It is also unclear whether there would be an advantage for intentional learning if all subjects had to answer an orienting question about the words.

Experiment 1 employed a somewhat unusual incidental-learning procedure, the digit-recall paradigm of maintenance rehearsal (Glenberg, Smith, \& Green, 1977; Rundus, 1977). This paradigm is an alteration of the classic Brown-Peterson short-term memory task. Subjects are told that they are in an experiment on short-term memory for digits. They are required to repeat words aloud as a distractor task between presentation and recall of a series of digits. After a number of trials, an unexpected test on memory for the words is given. In this task, subjects are motivated to process 
the words as shallowly as possible so that their attention may be maximized on the digits. This allows the experimenter greater control over the behavior of the subjects than would the typical incidental-learning task, in which there is no competing activity.

In Experiment 1, words occurred either once or twice. At the end of the experiment, the subjects were asked to indicate the frequency of the words. A test on the relative duration of the rehearsal interval for each word was also given. Each word was rehearsed for either 4 or $12 \mathrm{sec}$.

\section{EXPERIMENT 1}

\section{Method}

Subjects. Thirty-two people who responded to notices distributed around the Yale University campus during the summer participated. Each was tested individually and was paid $\$ 3.00$ after the 1 -h session had been completed.

Procedure. The crucial comparison here is between incidentallearning subjects and intentional-learning subjects. The incidentallearning subjects were told that they were in an experiment on short-term memory for digits and that they would have to repeat words aloud as a distractor activity between presentation and recall of the digits. They were told that they would not be tested on the words. The intentional-learning subjects were warned that, at the end of the experiment, their memory for the words would be tested. Since Shaughnessy (1981) had shown that subjects expecting a test often engage in nothing more than maintenance rehearsal anyway, the importance of doing well on the test of the words was stressed. One should note that even the subjects in the intentional-learning group were not told that they would be tested specifically on the frequency and the duration of the words.

Once the instructions had been given, the procedure was the same for both incidental- and intentional-learning groups. The subjects were seated in front of a computer terminal controlled by a TRS- 80 microprocessor. A trial began with a warning signal, a row of asterisks that appeared briefly in the center of the screen. One second after the asterisks had disappeared, a string of five digits appeared in the same location. The subjects were instructed to read the digits aloud once as soon as they had seen them. The digits disappeared after $1 \mathrm{sec}$. Then, a word was shown on the screen for 1 sec. After the word had disappeared, a single asterisk appeared and started flashing once per second. The subject was instructed to say the word aloud twice each time the asterisk flashed. The asterisk flashed for either 4 or $12 \mathrm{sec}$. After this rehearsal period had ended, the word "Recall" appeared on the screen, and the subject had to recall verbally the five-digit string shown at the beginning of the trial. After a $10-\mathrm{sec}$ intertrial interval, a new trial began.

In all, each subject went through 96 trials. The duration of the rehearsal interval was $4 \mathrm{sec}$ on half of the trials and $12 \mathrm{sec}$ on the other half. Of the 64 words that were used in the experiment, 32 appeared only once in a session, and the others occurred on two nonconsecutive trials. If a word occurred twice, it was always rehearsed for the same duration on both presentations. The number of trials between occurrences of a repeated item ranged from 2 to 19 trials, with a mean of 7.8 trials. The words were all common, four-letter nouns and were counterbalanced across subjects for both duration and frequency.

After all 96 trials had been completed, the subjects were asked to count backward from the number 200. After the subjects had counted for $1 \mathrm{~min}$, the experimenter began the tests on the duration and frequency of the words. The subjects were shown one pair of words at a time, each of which had appeared in the experiment. One of the words had been rehearsed for
$4 \mathrm{sec}$, and the other for $12 \mathrm{sec}$. Half of the subjects in each group had to choose the word that had been rehearsed for the longer interval; the other half had to say which word had been rehearsed for the short interval. (Since this manipulation had no effect on performance, it will not be discussed further.) Then the subjects had to decide the items' frequencies. Both members of each pair had occurred equally often: Either both had occurred on two separate trials or both had been shown only once. The subjects were asked to say whether both words had been shown once or both had been shown twice. (It should be kept in mind that the subjects were judging the frequency of a pair of items, one of which had been rehearsed for $4 \mathrm{sec}$ and the other for $12 \mathrm{sec}$; thus, the total duration was the same for all pairs.)

\section{Results and Discussion}

The mean proportions correct for the incidentallearning and intentional-learning groups on the frequencyjudgment, duration-judgment, and digit-recall tasks are shown in Table 1. Intentionality of learning had large effects on accuracy in the frequency-judgment and digitrecall tasks but had little, if any, effect on the accuracy of duration judgments.

Accuracy of frequency judgments. There was a significant difference between the groups in the accuracy of their judgments of frequency $[\mathrm{F}(1,30)=9.00, \mathrm{MSe}=$ 0.28 ]. This result seems inconsistent with the claim that frequency information is not affected by the intentionality of learning.

An alternative way of measuring the accuracy of frequency estimations is to calculate for each subject the correlation between true and estimated frequency. The mean correlations were .41 for incidental-learning subjects and .71 for intentional-learning subjects. This difference was easily significant $[F(1,30)=10.39, \mathrm{MSe}=$ $0.07]$.

The more common mistake $(67 \%$ of total errors for the incidental group and $61 \%$ for the intentional group) was saying that an item occurred once although it had really occurred twice. The difference in error patterns between the groups did not quite reach significance $[F(1.30)=3.81, \mathrm{MSe}=0.26, \mathrm{p}=.06]$. Since this large number of underestimations may only reflect a response bias, one should hesitate before trying to draw any substantive conclusions from it

Duration judgments. Accuracy of duration judgments for the rehearsal intervals of the words was not affected by intentionality of learning $[F(1,30)=0.15$, $\mathrm{MSe}=0.21]$. It might be claimed that performance was too close to chance levels on this task for any significant difference between the groups to be detected. I carried

Table 1

Mean Proportion Correct in Experiment 1

\begin{tabular}{lcc} 
& \multicolumn{2}{c}{ Group } \\
\hline \multicolumn{1}{c}{ Test } & Intentional & Incidental \\
\hline Frequency & .850 & .691 \\
Duration & .562 & .559 \\
Digit Recall & .674 & .873 \\
\hline
\end{tabular}


out another experiment, in which the duration intervals were 4 and $32 \mathrm{sec}$. Thirty-six subjects participated in this study. There were 72 trials in this session, with no item occurring more than once; otherwise, the procedure was the same as in Experiment 1. This raised performance somewhat (mean proportions correct were .61 for the incidental-learning group and .62 for the intentionallearning group), but there was still no difference between groups. Thus, there was no evidence that intentionality of learning has an effect on the accuracy of memory for duration.

Digit recall. The incidental-learning group recalled significantly more five-digit series correctly than did the intentional-learning group $[\mathrm{F}(1,30)=9.30$, MSe $=$ $306.60]$. This is not surprising, since the intentionallearning subjects were presumably devoting more effort to learning the words than were the incidental-learning subjects.

The duration of the rehearsal interval had no effect on recall of the digits. The mean proportions of digit sequences correctly recalled for 4 - and 12 -sec intervals were .87 and .87 for the incidental-leaming condition and .67 and .68 for the intentional-learning condition. This null effect of rehearsal interval on the recall of digit sequences has been found at times in other maintenancerehearsal experiments (e.g., Glenberg \& Adams, 1978; Glenberg \& Bradley, 1979; Glenberg et al., 1977).

\section{EXPERIMENT 2}

This experiment attempted to examine further the finding that intentionality of learning affected memory for frequency. There were three groups of subjects: One was led to believe that there would be no test on the words (incidental learning), another was told only that their "memory for the words will be tested" (intentional learning), and a third group was told explicitly that they would be tested on how often each word occurred (frequency learning).

Experiment 2 also tried to examine the cause of the difference between incidental and intentional learning in frequency estimation. It has been shown (e.g., Hyde \& Jenkins, 1969) that the difference in recall between intentional and incidental learning is greatly reduced when subjects have to perform an orienting task before each item. These tasks apparently serve to equalize the processing carried out by the subjects. Experiment 2 tested whether using orienting tasks for each word would decrease the difference between incidental and intentional learning of frequency. Before beginning rehearsal of each word, the subjects had to answer a question about it. The orienting questions involved either perceptual (Does this word contain a particular letter?) or semantic (Is this word a member of a particular category?) processing.

\section{Method}

Subjects. Fifty-four subjects were recruited in the same way as for Experiment $I$ and were paid $\$ 3.00$ each for their participation.

Procedure. There were several important differences between the procedure used in Experiment 2 and that followed in Experiment 1. There were three groups: incidental learning, intentional learning, and frequency learning. The frequencylearning group was warned that there would be a test on how often each word had occurred.

Each digit-recall trial was similar to the trials in Experiment 1, but with an important exception: The subjects had to answer an orienting question about each word. After the warning signal had been shown, either a letter of the alphabet or a category name (e.g., ANIMALS, FOODS) appeared on the screen briefly. Then the five-digit number was shown for 1 sec. After that, the tobe-rehearsed word appeared. As soon as the word had appeared, the subjects had to say "yes" or "no," indicating whether the rehearsal word contained that letter (if a letter had appeared earlier) or whether it was a member of that category (if a category name had been shown). The two orienting tasks occurred equally often. As soon as the subjects had performed the orienting task, they had to repeat the word aloud twice per second for $4 \mathrm{sec}$. Then a signal to recall the five-digit series came on.

Each subject was seated in front of a terminal controlled by a Northstar Horizon computer system. The experimenter read the appropriate instructions (incidental learning, intentional learning, or frequency learning). The subject then went through 98 digit-recall trials. The rehearsal duration for each trial was $4 \mathrm{sec}$. In all, 42 different words were shown, with equal numbers being shown on 1,2, or 4 trials and with each kind of orienting question. The number of items between occurrences of a repeated word ranged from 2 to 13 , with a mean of 6.39 items. If a word was repeated, the same kind of orienting question (letter search or category membership) was always asked, but not the exact same question.

After the subject had gone through 98 trials, he or she was asked to count backward from 200 for $1 \mathrm{~min}$. Then the frequency test began. All 42 items were shown 1 at a time, and the subject had to say whether the word had occurred one, two, or four times.

\section{Results and Discussion}

The results are shown in Table 2. There are two measures of accuracy reported for the frequency. judgment task. One is the proportion of items assigned the correct frequency. The other is the mean unsigned deviation, the average of the differences between the true frequency of an item and the subject's estimation. (Since only two frequencies were used in Experiment 1, these two measures were equivalent there.) Analyses

Table 2

Mean Performance in Experiment 2 on Letter-Search and Category-Verification Trials

\begin{tabular}{lccc}
\hline & \multicolumn{3}{c}{ Group } \\
\cline { 2 - 4 } & Frequency & Intentional & Incidental \\
\hline \multicolumn{4}{c}{ Frequency* } \\
Letter Trials & $.521(.704)$ & $.579(.700)$ & $.501(.828)$ \\
Category Trials & $.619(.612)$ & $.604(.624)$ & $.529(.799)$ \\
\multicolumn{5}{c}{ Digit Recall } \\
Letter Trials & .461 & .386 & .669 \\
Category Trials & .459 & .368 & .658 \\
\hline
\end{tabular}

*Numbers inside parentheses are mean unsigned deviations for frequency estimation. Numbers outside parentheses are mean proportions correct for frequency estimation and digit recall. 
of both measures yielded identical conclusions. Since the mean unsigned deviation contains the most information, only the analysis based on this measure will be discussed in detail. Performance on the orienting tasks was near $100 \%$ in all conditions, so no analysis of this dependent variable will be reported.

Accuracy of frequency judgments. If one looks at the mean accuracy for the three groups, it appears that the intentional-learning and frequency-learning groups were about equal and that both were more accurate than the incidental-learning group. The analysis of variance carried out on mean unsigned deviations confirmed this conclusion. There was a significant overall effect of instructional set $[F(2,51)=3.81]$. Planned contrasts showed that the intentional-learning group was more accurate than the incidental-learning group $[F(1,51)$ $=5.96]$, and that the frequency-learning group was also more accurate than the incidental-leaming group $[F(1,51)=5.44]$. However, the intentional-learning and frequency-learning groups did not differ significantly from each other $[F(1,51)=.12]$. (For all of the tests mentioned above, $\mathrm{MSe}=27.68$.)

The semantic orienting task (category membership) led to significantly greater accuracy in frequency estimation than did the perceptual task (letter search) $[F(1,51)=10.69]$. However, this variable did not interact with instructional set $[F(2,51)=0.57]$. (For both tests, $\mathrm{MSe}=22.07$.) This is reminiscent of the findings reported by Hyde and Jenkins (1969) for free recall. In that study, a semantic orienting question led to better recall than did a perceptual question, but the difference was equal for intentional- and incidental-learning subjects.

This pattern of results is also found if one measures accuracy by determining the correlation between true and estimated frequency for each subject. The mean correlations on letter-search and category-verification trials were .46 and .48 for the incidental-learning group, .52 and .66 for the intentional-learning group, and .52 and .62 for the frequency-learning group. There was a significant effect of instructional group $[\mathrm{F}(2,51)=3.35]$. The incidental-learning group was significantly less accurate than was either the intentional-learning group $[F(1,51)=6.06]$ or the frequency-learning $[F(1,51)=$ 3.81] group. However, the intentional-learning subjects did not differ significantly from the frequency-learning subjects $[F(1,51)=0.29]$. (For all of these tests, MSe = 0.05.) Type of orienting task had a significant effect on these correlations $[F(1,51)=6.72]$ but did not interact with instructional group $[F(1,51)=0.83]$. (In both cases, $\mathrm{MSe}=0.03$.)

In short, the finding reported in Experiment 1 that intentional learning of a list of words leads to better estimation of frequency than does incidental learning was replicated. The advantage for intentional-learning subjects was smaller than in Experiment 1. This probably was a result of the orienting tasks performed on the items prior to rehearsal; this sort of activity makes the processing carried out on each item more equal for both
Table 3

Mean Estimated Frequency in Experiment 2 as a Function of Instructional Group, Orienting Task, and True Frequency of the Item

\begin{tabular}{lccccccc} 
& \multicolumn{6}{c}{ Orienting Task } \\
\cline { 2 - 6 } & \multicolumn{6}{c}{ Letter Search } & \multicolumn{2}{c}{ Category Verification } \\
\cline { 2 - 7 } \cline { 5 - 7 } Group & 1 & 2 & 4 & 1 & 2 & 4 \\
\hline Frequency & 1.38 & 2.04 & 2.82 & 1.53 & 2.30 & 3.44 \\
Intentional & 1.33 & 1.65 & 2.63 & 1.33 & 1.90 & 3.15 \\
Incidental & 1.34 & 1.83 & 2.51 & 1.63 & 2.21 & 3.00 \\
\hline
\end{tabular}

groups and tends to reduce the advantage for intentional learning (see Hyde \& Jenkins, 1969). At the same time, it was also shown that instructing subjects specifically to learn frequency did not lead to higher accuracy than did simply telling them to learn the words; this null finding has been reported by previous investigators (Flexser \& Bower, 1975; Howell, 1973; Zacks et al., 1982, Experiment 3).

Frequency estimates. It is also possible to see how instructional set and orienting task affected frequency estimates. The mean estimated frequencies are shown in Table 3. There was a significant effect of instructional set $[\mathrm{F}(2,51)=3.33, \mathrm{MSe}=0.06]$ and a significant interaction between instructional set and frequency $[F(2,51)=3.46$, MSe $=0.18]$. These effects seem to be due primarily to a tendency by the intentional-learning subjects to give particularly low frequency estimates, especially for items that really had occurred once or twice.

Orienting task also affected frequency estimation $[\mathrm{F}(1,51)=50.51, \mathrm{MSe}=0.18]$, with the category. verification task leading to larger frequency estimations. Orienting task interacted with true frequency $[F(2,102)$ $=6.53, \mathrm{MSe}=0.17]$, with the effect of orienting task being greatest at the higher true frequencies.

Digit recall. The mean proportion of five-digit sequences correctly recalled in each of the conditions in Experiment 2 is shown in Table 2. As in Experiment 1, there was a significant effect of instructional set $[\mathrm{F}(2,51)$ $=9.82]$. The incidental-learning group recalled more sequences correctly than did either the intentional-learning group $[F(1,51)=18.76]$ or the frequency-learning group $[F(1,51)=8.84]$. The intentional-and frequency-learning groups did not differ significantly from each other $[F(1,51)=1.84] .($ For the above tests, $\mathrm{MSe}=215.99$.)

Type of orienting task did not have a significant effect on digit recall $[F(1,51)=2.31]$ and did not interact with intentionality of learning $[\mathrm{F}(2,51)=0.60]$. (For the above tests, $\mathrm{MSe}=9.16$.)

\section{GENERAL DISCUSSION}

The results from these experiments seem clear. Instructional set and intentionality of learning do have an 
effect on memory for frequency. People who are led to believe that they will not be tested on a list of words are less accurate in an absolute-frequency test than are those who expect to be tested. As had been found by previous investigators (Flexser \& Bower, 1975; Howell, 1973; Zacks et al., 1982), accuracy was not affected by whether the subjects were told to expect a frequency test or only an unspecified test.

The results here do not prove that frequency is not encoded automatically. In fact, it is quite possible that frequency is encoded equally well under conditions of incidental and intentional learning but that this information is more easily retrieved when subjects are intentionally learning the items. Although retrieval was not discussed by Hasher and Zacks (1979), it must determine, in part, performance on a frequency-estimation test. No one has ever claimed that retrieval of frequency information is automatic. Thus, the fact that intentionality of learning affects frequency estimation cannot prove that frequency information is not encoded automatically because it is not clear that this effect is due to encoding processes. However, if it had been found that intentionality of learning had no effect, this would not have necessarily meant that frequency is encoded automatically. Rather, it might simply mean that intentionality has opposite effects on the encoding and retrieval stages and that these effects tend to cancel each other out. In other words, it is impossible to determine automaticity of encoding by looking at the effects of intentionality.

It could be claimed that encoding of frequency information should be considered automatic if it meets the other four criteria proposed by Hasher and Zacks (1979). However, these other criteria also do not separate encoding from retrieval factors, so one cannot interpret them unambiguously. Moreover, the evidence regarding the other criteria is not very strong. To illustrate this, I will briefly review this evidence.

\section{Interference Between Operations}

According to Hasher and Zacks (1979), there should be no interference between remembering frequency and performing other operations. The only relevant study comes from Zacks et al. (1982, Experiment 3). Groups of subjects were told to expect a frequency test, a freerecall test, or both. At the end of the experiment, subjects were given either a frequency or a free-recall test. Subjects who were told to expect both tests did as well on the frequency test as those who were expecting only the frequency test. Zacks et al. interpreted this finding as supporting the notion that there is no interference between encoding frequency and encoding other sorts of information. However, to meet this criterion, the relationship has to be symmetric: Not only must frequency encoding be unimpaired by other activities, but also performance on these other tasks must not suffer as a result of frequency encoding. This criterion is not met. Subjects who were expecting to be given both recall and frequency tests recalled fewer items than did those who were expecting only recall. It is as if trying to learn frequency interfered with preparation for free recall.

\section{Alterations in Attentional Capacity}

Another of Hasher and Zacks's (1979) criteria is that frequency encoding should not be affected by the attentional capacity available to the subject. The only relevant study was performed by Hasher and Zacks (1979, Experiment 3). This study compared accuracy in frequency estimation between depressed and nondepressed people. Subjects were given a paper-and-pencil variant of the Beck Depression Inventory and were assigned to either the depressed or the nondepressed group, the median being used as the criterion. Then they participated in a frequency-estimation experiment. There was no significant difference between the groups.

Unfortunately, there are many serious problems with Hasher and Zacks's (1979) Experiment 3. For example, there is no reliability reported for the variant of the Beck Depression Inventory that was used. Also, using a median split to assign subjects to groups maximizes the likelihood of misclassification; a correlational analysis would have been a much more sensitive way to analyze these data. Only 16 subjects (8 in each group) were run, an extremely small sample size on which to base a null result. Finally, it is unclear whether depression is really an appropriate way to study cognitive capacity for tasks of this sort.

\section{Developmental Trends}

Another of Hasher and Zacks's (1979) criteria for automatic encoding is that no developmental trends should be found. Here, the evidence is mixed, with several studies failing to show any trends (Hasher \& Chromiak, 1977; Hasher \& Zacks, 1979, Experiment 1) but with at least one study (Ghatala \& Levin, 1973) finding improvement in accuracy as a function of age. In this study, the trend was particularly strong when pictures were used as stimuli. More research is needed to determine the circumstances under which developmental trends in memory for frequency become evident.

\section{Practice Effects}

The remaining criterion of Hasher and Zacks (1979) is that accuracy should not improve as a function of practice. It has been shown that accuracy on a frequencyestimation task does not improve after having studied frequency on one (Hasher \& Chromiak, 1977, Experiment 2) or three (Zacks et al., Experiments 1 and 2) previous lists. Thus, this criterion has been met in the experiments that have been carried out so far. Whether extended practice (e.g., practice over several sessions) would also have no effect has not been studied.

\section{Conclusions}

In short, we see that there is not strong evidence that frequency encoding meets the criteria suggested by Hasher and Zacks (1979) for automaticity. This paper 
has reported two experiments that show that intentionality of learning does affect frequency performance. A review of the literature suggests that the evidence regarding at least three of the other four criteria is either negative or unclear. Moreover, it should be noted that none of these criteria directly measures encoding; whatever results have been found in these studies may be due to retrieval, rather than encoding, processes. Therefore, these criteria can never be used with confidence to determine whether frequency (or any other sort of information) is encoded automatically. This suggests that automaticity of encoding may be impossible to determine in situations in which retrieval and decision processes are not controlled.

\section{REFERENCES}

Flexser, A. J., \& Bower, G. H. Further evidence regarding instructional effects on frequency judgments. Bulletin of the Psychonomic Society, 1975, 6, 321-324.

Ghatala, E. S., \& Levin, J. L. Developmental differences in frequency judgments of words and pictures. Journal of Experimental Child Psychology, 1973, 16, $495-507$.

Glenbera, A., \& Adams, F. Type 1 rehearsal and recognition. Journal of Verbal Learning and Verbal Behavior, 1978, 17, $455-463$.

Glenberg, A., \& Bradiey, M. M. Mental contiguity. Journal of Experimental Psychology: Human Learning and Memory, 1979, 5, 88-97.

Glenberg, A., Smith, S. M., \& Green, C. Type 1 rehearsal:
Maintenance and more. Journal of Verbal Learning and Verbal Behavior, 1977, 16, 339-352.

Hasher, L., \& Chromiak, W. The processing of frequency information: An automatic mechanism? Journal of Verbal Learning and Verbal Behavior, 1977, 16, 173-184.

HASHER, L., \& ZACKs, R. T. Automatic and effortful processes in memory. Journal of Experimental Psychology: General, 1979, 108, 356-388.

Howell, W. C. Storage of events and event frequencies: A comparison of two paradigms in memory. Journal of Experimental Psychology, 1973, 98, 260-263.

Hyde, T. S., \& Jenxins, J. J. Differential effects of incidental tasks on the organization of recall of a list. Journal of Experimental Psychology, 1969, 82, 472-481.

LEHMAN, E. B. Memory for modality: Evidence for an automatic process. Memory \& Cognition, 1982, 10, 354-564.

Mandler, J. M., Seegmiller, D., \& DaY, J. On the coding of spatial information. Memory \& Cognition, 1977, 5, 10-16.

Rose, R. J., \& Rowe, E. J. Effects of orienting task and spacing of repetitions on frequency judgments. Journal of Experimental Psychology: Human Learning and Memory, 1976, 2, 142-152.

Rundus, D. Maintenance rehearsal and single-level processing. Journal of Verbal Learning and Verbal Behavior, 1977, 16, 665-681.

ShaUghNessy, J. J. Memory monitoring accuracy and modification of rehearsal strategies. Journal of Verbal Learning and Verbal Behavior, 1981, 20, 216-230.

Zacks, R. T., Hasher, L., \& SANFt, H. Automatic encoding of event frequency: Further findings. Journal of Experimental Psychology: Learning, Memory, and Cognition, 1982, 8, 106-116.

(Manuscript received March 8, 1983; revision accepted for publication September 13, 1983.) 